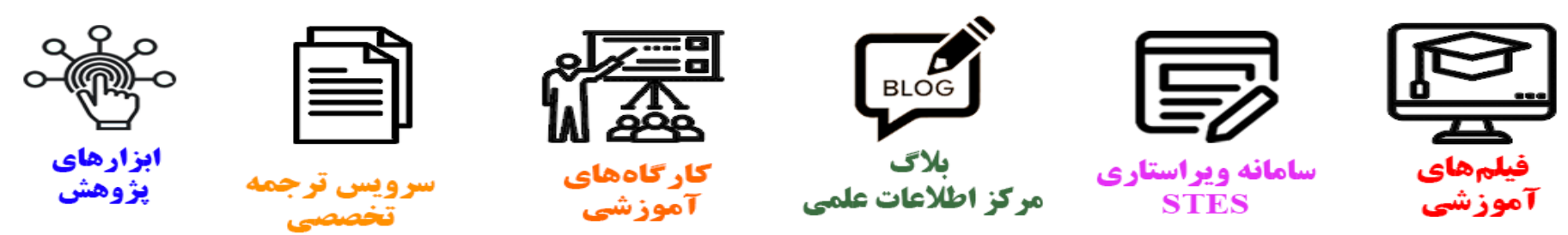

\title{
(c)
}

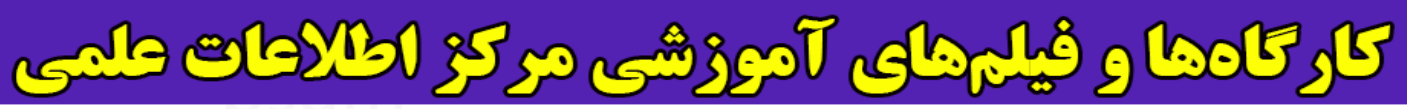
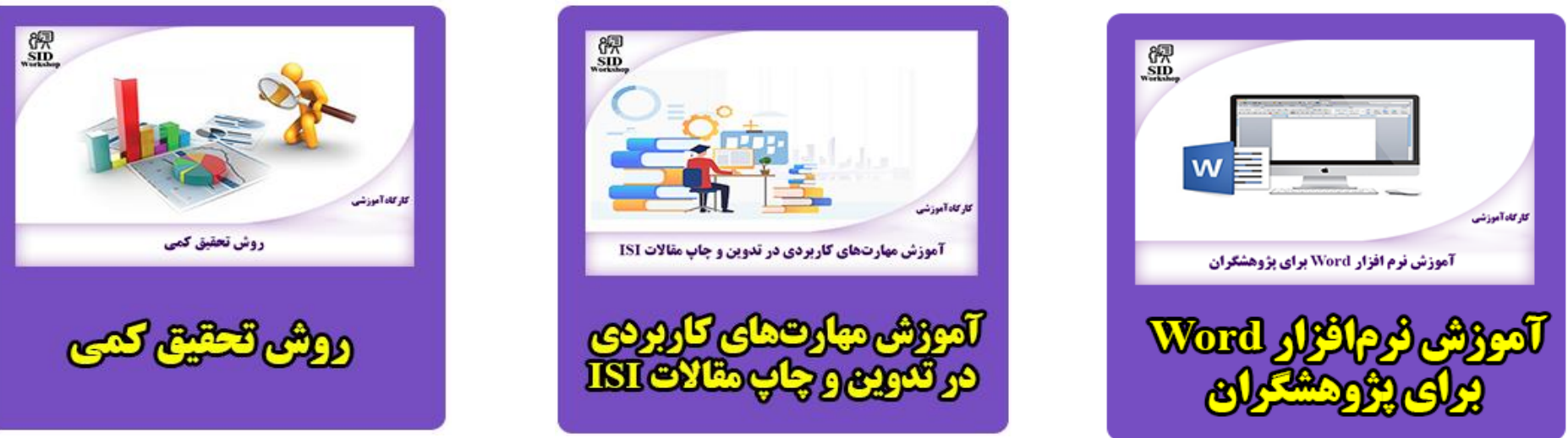


\title{
International Journal of Engineering
}

Journal Hom e page: ww w. ije.ir

\section{Detection of Alzheimer's Disease Using Multitracer Positron Emission Tomography Imaging}

\author{
H. Dehghan, A. A. Pouyan, H. Hassanpour* \\ School of Computer Engineering and Information Technology, Shahrood University of Technology, Shahrood, Iran
}

\section{PAPER INFO}

\section{Paper history:}

Received 17 March 2013

Accepted in revised form 20 June 2013

\section{Keywords:}

Alzheimer's Disease

Multitracer PET

Neuroimaging

Principal Component Analysis

\begin{abstract}
A $B$ S S T T R A A C
Alzheimer's disease is characterized by impaired glucose metabolism and demonstration of amyloid plaques. Individual positron emission tomography tracers may reveal specific signs of pathology that is not readily apparent on inspection of another one. Combination of multitracer positron emission tomography imaging yields promising results. In this paper, 57 Alzheimer's disease neuroimaging initiative subjects that had FDG and PiB-positron emission tomography neuroimaging scans at the same time were used for development of proposed multitracer classification method. The subject's brain image was automatically parcellated into 48 pre-defined regions of interest. Then, 96 features were extracted for each subject. The principal features weere extracted using principal component analysis, then they were combined based on intersection strategy. Finally, a support vector machine was adopted to evaluate the classification accuracy. Combination of two tracers with positron emission tomography scan yielded a higher diagnostic accuracy in Alzheimer's disease compared to individual tracer and other combination methods.
\end{abstract}

\section{INTRODUCTION}

Alzheimer's disease (AD) is the most common cause of dementia [1-3]. It is a progressive neurodegenerative disorder that impairs memory, cognitive function, thinking, behavior and language [4, 5]. It has been reported that 35.6 million people worldwide are suffering from dementia and this number will increase to 65.7 million by $2030[6,7]$. There is still no cure for the disease but early detection of $\mathrm{AD}$ is a prerequisite for early treatment [8].

The neuroimaging is an approach increasingly used for accurate clinical diagnosis of $\mathrm{AD}[9,10]$. There are two main categories of neuroimaging: structural neuroimaging (e.g. magnetic resonance imaging (MRI)) and functional neuroimaging (e.g. positron emission tomography (PET)) [10]. Although the brain atrophy measured using MRI in patient at prodromal stage of the $\mathrm{AD}$ (mild cognitive impairment (MCI)) is similar to those in normal elderly people, PET has the ability to display very mild symptoms such as amyloid plaques and impaired glucose metabolism, which are signed

${ }^{*}$ Corresponding Author Email: h.hassanpour@shahroodut.ac.ir (H. Hassanpour) with AD [11, 12]. Therefore, extracting the features from brain regions using PET neuroimaging can provide useful hallmarks for discriminant normal control (NC) from $\mathrm{AD}$ (or $\mathrm{MCI}$ ). Brain glucose metabolism and amyloid plaques are measured by $18 \mathrm{~F}$-fluorodeoxy (FDG) and 11C Pittsburgh Compound-B (PiB) tracers with PET scans, respectively [10, 13]. Nevertheless, PET neuroimaging provides specific information about subjects with and without $\mathrm{AD}$. In this paper, multitracer $\mathrm{PiB}$ and FDG-PET neuroimaging that has been acquired at the same time are combined. These can provide valuable information, including amyloid plaque and glucose metabolism. The problem of classification in case of multiple feature space obtained from different sources is combinations of individual classifiers or combination of feature spaces [14]. The aim of combination of feature spaces is to obtain highly accurate classifiers by combining individual feature space. As the most common way, all features derived from different data sources, i.e. modalities, are concatenated into a larger feature space. Other ways for combination feature spaces can be grouped into two basic categories: 1) ad hoc methods [15], and kernel combination methods $[16,17]$. 
TABLE 1. Demographic characteristics of the studied population (from the ADNI database).

\begin{tabular}{|c|c|c|c|c|c|c|}
\hline \multirow{2}{*}{ Group } & \multirow{2}{*}{ Number } & \multirow{2}{*}{$\begin{array}{c}\text { Gender } \\
(\mathrm{M} / \mathrm{F})\end{array}$} & \multicolumn{2}{|l|}{ Age } & \multicolumn{2}{|c|}{ MMSE } \\
\hline & & & Mean \pm SD & Range & Mean \pm SD & Range \\
\hline $\mathrm{NC}$ & 19 & $11 / 8$ & $78.8 \pm 5.5$ & $71-87$ & $28.5 \pm 1.5$ & $24-30$ \\
\hline MCI & 20 & $15 / 5$ & $76.6 \pm 8.0$ & $61-90$ & $26.1 \pm 2.8$ & $20-30$ \\
\hline $\mathrm{AD}$ & 18 & $11 / 7$ & $73.6 \pm 8.6$ & $55-88$ & $20.2 \pm 4.2$ & $12-26$ \\
\hline
\end{tabular}

MRI, FDG-PET and also CSF modalities were combined based on multi-kernel combination for both $\mathrm{AD}$ and MCI classifications [18]. The proposed method was operated within support vector machine (SVM) learning classifier. However, in this paper an approach for combing feature spaces derived from FDG and PIBPET modalities has been proposed and compared with multi-kernel SVM method used in [18].

\section{SUBJECTS}

Data used in this research were obtained from the Alzheimer's disease Neuroimaging Initiative (ADNI) database (http://www.loni.ucla.edu/ADNI). In this study, 57 subjects, including $18 \mathrm{AD}$ patients, $20 \mathrm{MCI}$ patients, and 19 normal controls (NC) were collected from the ADNI public database (LONI, University of California, http://www.loni.ucla.edu/ADNI/Data). All patients and healthy subjects (subjects were manually found between all ADNI databases) had [11C] PIB-PET and [18F] FDG-PET imaging. A detailed description of PET protocol and acquisition can be found at "www.adni-info.org". The demographic characteristics of the subjects used in this study have been summarized in Table 1.

\section{METHOD}

3. 1. Preprocessing All PET images have been preprocessed with SPM8 [19] (Wellcome Department of Cognitive Neurology, Institute of Neurology, London, www.fil.ion.ucl.ac.uk/spm) and was performed in MATLAB 7.10.0 (MathWorks Inc., Sherborn, MA). Preprocessing was performed in the following steps:

- Normalization This step includes registration and normalization into a standardized coordinate system. The goal of normalization is to register images from different participants into a rough template image. Normalization was performed to ensure that the voxels in different images refer to the same anatomical positions in the brain [20].
Each individual subject's FDG-PET was automatically spatially normalized to the PET Montreal Neurological Imaging (MNI) template (http://www.bic.mni.mcgill.ca) through 12parameters affine model [21] using the SPM8 software with the default settings. PiB-PET images were also normalized to the template generated by averaging all normal control images. After the normalization step, the dimensions of the obtained images were re-sampled to a voxel spacing of 2 $\mathrm{mm} 3$ and size $91 \times 109 \times 91$ voxels.

- Smoothing Smoothing is used to reduce the high frequency noise and increase the signal-to-noise ratio of the hemodynamic effect. Smoothing was performed using a Gaussian kernel with full-width of half-maximum (FWHM) of $8 \mathrm{~mm}$.

3. 2. Features In this study, features were obtained by voxel grouping using a labeled atlas. Atlas-based approach used for PET images is similar to method used in [18]. After pre-processing, the subject's brain image was automatically parcellated into 48 pre-defined regions of interest (ROI). The anatomical regions were constructed based on the Harvard-Oxford probabilistic atlas that distributed with FMRIB Software Library (FSL: $\quad$ http://www.fmrib.ox.ac.uk/fsl/fslview/atlasdescriptions.html). The Harvard-Oxford atlas includes 48 regions.

Feature used in this study was obtained using averaging all voxels intensity of each ROI region. Previously, Zhang in [18] combined MRI and FDG-PET modalities as a multimodal classification of Alzheimer's disease. For each MRI and FDG-PET image, they extracted 93 features from 93 automatically labeled ROIs [22] by calculating the average intensity of each ROI region. Unlike [18], used 93 ROIs, that developed by Kabani at the MNI [22], we parcellated images into 48 ROIs based on the Harvard-Oxford atlas.

3. 3. Feature selection/extraction: PCA Principal component analysis (PCA) is one of the most effective methods in data compression and pattern recognition. The aim of PCA is to reduce the dimension of the data. PCA is used to omit redundant data for feature selection and feature extraction. Since PCA works in linear domain, it is used in linear applications, such as, signal processing, image processing, system and control theory and communication.

PCA depends on eigenvector method designed to model linear variations in large dimensional data. PCA performs dimension reduction by projecting the original $\mathrm{n}$-dimensional data to $\mathrm{k}$-dimensional $(\mathrm{k}<<\mathrm{n})$ linear subspace [23].

3. 4. Support Vector Machine Support Vector Machine (SVM) creates an optimal hyper plane using binary labeled training data by maximizing the margin 
between two classes [24]. Once the model has been trained, the measures of the diagnostic accuracy can be carried out with the test set. A detailed description of SVM algorithms can be found in [25-27]. The idea behind SVMs is to map the input patterns into a highdimensional feature space through a suitable kernel function. The mapping is done in order to make the classification problem simpler in the high-dimensional feature space [28]. In this paper, the SVM classifier was implemented using libsvm toolbox available at http://www.csie.ntu.edu.tw/cjlin/libsvm in MATLAB 7.10.0 (MathWorks Inc., Sherborn, MA).

The reason of wide application of SVM in AD domain is that SVM often performs more efficiently than other classifiers $[2,29,30]$.

3. 4. Proposed Method The proposed approach in this research involves two major steps: training step; learning the useful information, and testing step. Firstly, some subjects were used for training step. Training was achieved by means of the leave-one-out method, a technique that iteratively holds out one subject for test [31]. After the optimum parameters were obtained with the remaining subjects, in the test step, each new subject that was left out was assigned to its appropriate class.

In order to combine information derived from different images there are several ways in machine learning research. The most common way is to combine all features derived from different modalities into a longer feature vector [32]. Additionally, several researchers $[18,33,34]$ provide an alternative way by using a kernel combination to integrate different modalities for AD study. More detailed description for multi-kernel approach used in SVM can be found in [18]. In order to evaluate the proposed method, two methods proposed in [32] and [18] were implemented and compared with proposed method.

In this paper, a new approach for combination of the feature spaces derived from different neuroimaging scans, is proposed. First, the principal features are selected from two tracer image scans using PCA. Combining features that have been chosen in first step can achieve premier features, e.g. ROIs, of two tracers. In order to combine the features chosen from individual PET tracer image, there are two different methods, union and intersection.

Intersection method selects the repeated features selected in two of the PET tracer image by PCA. On the other hand, the results of union combination method are based on all features that have been selected by each of the two PET tracer images. Therefore, features extracted from each individual tracer image are combined and evaluated agaist the classification accuracy in discriminate $\mathrm{NC}$ and $\mathrm{AD}$ (or $\mathrm{MCI}$ ). It is based on premier features selected by intersection combination method.

\section{RESULTS}

In order to compare the performance of FDG-PET and PiB-PET images in AD, we perform experiments (1) using only each individual modality, FDG-PET and PiB-PET modalities, (2) combining features using multi-kernel and concatenation methods, and finally (3), using the proposed method. For posterior analysis, all subjects were arranged into two different groups, and the performance of our classification methods is tested in identification:

- NC vs. AD: NC subjects from AD patients,

- NC vs. MCI: NC subjects from MCI patients. The classification results are summarized in Tables 2 and 3. As discussed in Section 3, all experiments were carried out by the leave-one-out cross-validation method. The classification results are described in terms of accuracy, sensitivity and specificity values, the most widely statistics used in diagnostic test. For analysis of PET tracers each image modality of each subject was first parcellated into 48 ROIs using the Harvard-Oxford atlas. Afterward, all 48 ROIs were used in order to create the feature set by averaging the intensity value of voxels in each ROI. Using single-modality, FDG-PET reached highest accuracy rates. FDG-PET distinguished NC from AD (MCI) patients with 88.89\% (75\%), $89.74 \%$ (78.95) and $89.19 \%$ (76.92) for sensitivity, specificity and accuracy, respectively. In order to compare multi-kernel SVM method and concatenation method for combining features obtained from different PET tracers, we implemented these two methods. Table 2 shows the performance of these two combination methods. In all groups, combining features using multikernel method reached highest accuracy rates. For NC vs. AD, the multi-kernel SVM and concatenation SVM methods reached the accuracy of $83.78 \%$ and $88.24 \%$, respectively. For NC vs. MCI, multi-kernel method also reached higher accuracy $(87.5 \%)$ than those based on the concatenation $(76.92 \%)$. The results of this study show that the multi-kernel method achieved higher performance than concatenation methods in discriminanting between $\mathrm{NC}$ and $\mathrm{AD}$ (or MCI). The experimental results obtained in this research shows that considering the multi-kernel method is more accurate than concatenation method in combining different modalities. Moreover, multi-kernel method has advantages over combining different modalities [18]: 1) it provides a unified way to combine heterogeneous data when a different type of data cannot be directly concatenated, and 2) it offers more flexibility by using different weights on biomarkers of different modalities.

Multimodal imaging in AD has been previously studied by several researches $[18,24,25]$. As the most recent work, ([18]) evaluated MRI, FDG-PET and also $\mathrm{CSF}$, and combination of these modalities based on multi-kernel combination for both AD classification and 
TABLE 2. Classification results.

\begin{tabular}{|c|c|c|c|c|c|c|}
\hline \multirow{2}{*}{ Methods } & \multicolumn{3}{|c|}{ NC vs. AD (\%) } & \multicolumn{3}{|c|}{ NC vs. MCI (\%) } \\
\hline & Specificity & Sensitivity & Accuracy & Specificity & Sensitivity & Accuracy \\
\hline (1) FDG-PET & 78.947 & 88.888 & 83.78 & 78.95 & 65 & 71.79 \\
\hline (1) PIB-PET & 68.42 & 77.78 & 72.97 & 68.42 & 85 & 76.92 \\
\hline (2) Concatenation Method & 89.47 & 77.78 & 83.78 & 78.95 & 75 & 76.92 \\
\hline (2) Multi-Kernel Method & 94.12 & 82.35 & 88.24 & 78.95 & 80 & 79.49 \\
\hline (3) Proposed Method & 100 & 94.12 & 94.12 & 78.95 & 85 & 82.05 \\
\hline
\end{tabular}

TABLE 3. Comparison between performances reported in [35] and this study.

\begin{tabular}{|c|c|c|c|c|c|}
\hline Method & & Subjects & Specificity (\%) & Sensitivity (\%) & Accuracy (\%) \\
\hline \multirow[t]{2}{*}{ Zhang, D., et al [31] } & NC vs. AD (\%) & $52 \mathrm{NC}, 51 \mathrm{AD}$ & 93.3 & 93 & 93.2 \\
\hline & NC vs. MCI (\%) & $52 \mathrm{NC}, 99 \mathrm{MCI}$ & 66.0 & 81.8 & 76.4 \\
\hline \multirow[t]{2}{*}{ Proposed Method } & NC vs. AD (\%) & $17 \mathrm{NC}, 17 \mathrm{AD}$ & 100 & 94.12 & 94.12 \\
\hline & NC vs. MCI (\%) & $17 \mathrm{NC}, 20 \mathrm{MCI}$ & 78.95 & 85 & 82.05 \\
\hline
\end{tabular}

MCI classification. In this paper, we used a more significant feature selection/extraction method using PCA and combined features extracted from FDG-PET and PiB-PET image scans. Preliminary experimental results of individual modality as shown in proposed method for combination of FDG-PET and PIB-PET achieved higher accuracies compared to the approach reported in [18] (see Table 3). Performance rate reported in [18] for discriminanating between $\mathrm{NC}$ and MCI was $76.4 \%$ and our accuracy value is $82.05 \%$.

\section{CONCLUSION}

In this research, we combined different neuroimaging modalities, FDG-PET and PiB-PET, in the same group of $\mathrm{AD}$ (and $\mathrm{MCI}$ ) patients and normal controls. As individual neuroimaging technologies do not provide optimal information for the analysis pathology in $\mathrm{AD}$, combining neuroimaging modalities could provide higher recognition rate in $\mathrm{AD}$. Therefore, we evaluated individual PET images, and also combination of multitracer PET neuroimaging in AD. In order to evaluate the combinations of tracers with PET, we compared proposed method with concatenation and multi-kernel methods. This paper compares FDG-PET and PIB-PET modalities in order to discriminate NC from $\mathrm{AD}$ (or $\mathrm{MCI}$ ). We proposed a novel method for combining these tracers with PET neuroimaging scan and we compared concatenation and multi-kernel methods.

\section{REFERENCES}

1. SR., G., "Is it alzheimer's disease?", Postgrad Med, Vol. 101, (1997), 42-43.

2. Folstein, M. F., "Differential diagnosis of dementia: The clinical process", Psychiatric Clinics of North America, Vol. 20, No. 1, (1997), 45-57.

3. Morris, J. C., "Differential diagnosis of alzheimer's disease", Clinics in Geriatric Medicine, Vol. 10, No. 2, (1994), 257.

4. Petrella, J. R., Coleman, R. E. and Doraiswamy, P. M., "Neuroimaging and early diagnosis of alzheimer disease: A look to the future1", Radiology, Vol. 226, No. 2, (2003), 315-336.

5. López, M., Ramírez, J., Górriz, J. M., Álvarez, I., SalasGonzalez, D., Segovia, F., Chaves, R., Padilla, P., and GómezRío, M., "Principal component analysis-based techniques and supervised classification schemes for the early detection of alzheimer's disease", Neurocomputing, Vol. 74, No. 8, (2011), 1260-1271.

6. Mebane-Sims, I., "2009 alzheimer's disease facts and figures", Alzheimer's \& Dementia, (2009).

7. Prince, M. and Jackson, J., "World alzheimer report 2009", Alzheimer's Disease International, (2009).

8. Nordberg, A., "Pet imaging of amyloid in alzheimer's disease", The Lancet Neurology, Vol. 3, No. 9, (2004), 519-527.

9. Apostolova, L. G. and Thompson, P. M., "Mapping progressive brain structural changes in early alzheimer's disease and mild cognitive impairment", Neuropsychologia, Vol. 46, No. 6, (2008), 1597-1612.

10. Mueller, S. G., Weiner, M. W., Thal, L. J., Petersen, R. C., Jack, C. R., Jagust, W., Trojanowski, J. Q., Toga, A. W., and Beckett, L., "Ways toward an early diagnosis in alzheimer's disease: The alzheimer's disease neuroimaging initiative (adni)", Alzheimer's and Dementia, Vol. 1, No. 1, (2005), 55-66.

11. Braak, H. and Braak, E., "Staging of alzheimer's disease-related neurofibrillary changes", Neurobiology of Aging, Vol. 16, No. 3, (1995), 271-278. 
12. Delacourte, A., David, J., Sergeant, N., Buee, L., Wattez, A., Vermersch, P., Ghozali, F., Fallet-Bianco, C., Pasquier, F., and Lebert, F., "The biochemical pathway of neurofibrillary degeneration in aging and alzheimer's disease", Neurology, Vol. 52, No. 6, (1999), 1158-1158.

13. Nordberg, A., "Amyloid imaging in alzheimer's disease", Neuropsychologia, Vol. 46, No. 6, (2008), 1636-1641.

14. Damoulas, T. and Girolami, M. A., "Combining feature spaces for classification", Pattern Recognition, Vol. 42, No. 11, (2009), 2671-2683.

15. Berger, J. O., "Statistical decision theory and bayesian analysis", Springer, (1985).

16. Lee, W.-J., Verzakov, S. and Duin, R. P., Kernel combination versus classifier combination, in Multiple classifier systems., Springer. (2007). 22-31

17. Sonnenburg, S., Rätsch, G. and Schäfer, C., "A general and efficient multiple kernel learning algorithm", (2006).

18. Zhang, D., Wang, Y., Zhou, L., Yuan, H. and Shen, D., "Multimodal classification of alzheimer's disease and mild cognitive impairment", Neuroimage, Vol. 55, No. 3, (2011), 856-867.

19. Friston, K. J., Ashburner, J. T., Kiebel, S. J., Nichols, T. E. and Penny, W. D., "Statistical parametric mapping: The analysis of functional brain images: The analysis of functional brain images", Academic Press, (2011).

20. Ramírez, J., Górriz, J., Segovia, F., Chaves, R., Salas-Gonzalez D., López, M., Álvarez, I., and Padilla, P., "Computer aided diagnosis system for the alzheimer's disease based on partial least squares and random forest spect image classification", Neuroscience Letters, Vol. 472, No. 2, (2010), 99-103.

21. Woods, R. P., Grafton, S. T., Holmes, C. J., Cherry, S. R. and Mazziotta, J. C., "Automated image registration: I. General methods and intrasubject, intramodality validation", Journal of Computer Assisted Tomography, Vol. 22, No. 1, (1998), 139152.

22. Kabani, N. J., "3d anatomical atlas of the human brain", Neuroimage, Vol. 7, No., (1998), P-0717.

23. In Cowan, J., Tesauro, G. and Alspector, J., "Fast non-linear dimension reduction", Vol., No.

24. Rizk-Jackson, A., Stoffers, D., Sheldon, S., Kuperman, J., Dale, A., Goldstein, J., Corey-Bloom, J., Poldrack, R. A., and Aron, A R., "Evaluating imaging biomarkers for neurodegeneration in pre-symptomatic huntington's disease using machine learning techniques", Neuroimage, Vol. 56, No. 2, (2011), 788-796.
25. Luts, J., Ojeda, F., Van de Plas, R., De Moor, B., Van Huffel, S., and Suykens, J. A., "A tutorial on support vector machine-based methods for classification problems in chemometrics", Analytica Chimica Acta, Vol. 665, No. 2, (2010), 129-145.

26. Sanchez, A. and David, V., "Advanced support vector machines and kernel methods", Neurocomputing, Vol. 55, No. 1, (2003), $5-20$.

27. Schölkopf, B. and Smola, A., Learning with kernels., MIT Press, Cambridge, MA. (2002)

28. Vapnik, V., "The nature of statistical learning theory", Springer, (2000).

29. Magnin, B., Mesrob, L., Kinkingnéhun, S., Pélégrini-Issac, M., Colliot, O., Sarazin, M., Dubois, B., Lehéricy, S., and Benali, H., "Support vector machine-based classification of alzheimer's disease from whole-brain anatomical mri", Neuroradiology, Vol. 51, No. 2, (2009), 73-83.

30. Górriz, J., Ramírez, J., Lassl, A., Salas-Gonzalez, D., Lang, E., Puntonet, C., Álvarez, I., López, M., and Gómez-Río, M., "Automatic computer aided diagnosis tool using componentbased svm", in Nuclear Science Symposium Conference Record, 2008. NSS'08. IEEE, (2008), 4392-4395.

31. Illán, I., Górriz, J., Ramírez, J., Salas-Gonzalez, D., López, M., Segovia, F., Chaves, R., Gómez-Rio, M., and Puntonet, C. G., " 18 F-FDG pet imaging analysis for computer aided alzheimer's diagnosis", Information Sciences, Vol. 181, No. 4, (2011), 903916.

32. Dukart, J., Mueller, K., Horstmann, A., Barthel, H., Möller, H. E., Villringer, A., Sabri, O., and Schroeter, M. L., "Combined evaluation of fdg-pet and mri improves detection and differentiation of dementia", PLoS One, Vol. 6, No. 3, (2011).

33. Hinrichs, C., Singh, V., Xu, G. and Johnson, S. C., "Predictive markers for ad in a multi-modality framework: An analysis of mci progression in the adni population", Neuroimage, Vol. 55 , No. 2, (2011), 574-589.

34. Hinrichs, C., Singh, V., Xu, G. and Johnson, S., Mkl for robust multi-modality ad classification, in Medical image computing and computer-assisted intervention-miccai, Springer, (2009). 786-794.

35. M.M. López, J. Ramírez, J. M. Górriz, I. Álvarez, D. SalasGonzalez, F. Segovia, R. Chaves, "SVM-based CAD system for early detection of the Alzheimer's disease using kernel PCA and LDA", Neuroscience Letters, Vol. 464, (2009) 233-238 . 


\title{
Detection of Alzheimer's Disease Using Multitracer Positron Emission Tomography Imaging
}

\author{
H. Dehghan, A. A. Pouyan, H. Hassanpour
}

School of Computer Engineering and Information Technology, Shahrood University of Technology, Shahrood, Iran

$P A P E R \quad I N F O$

Paper history:

Received 17 March 2013

Accepted in revised form 20 June 2013

\section{Keywords:}

Alzheimer's Disease

Multitracer PET

Neuroimaging

Principal Component Analysis
جكيده

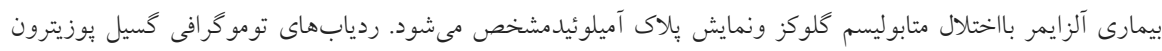

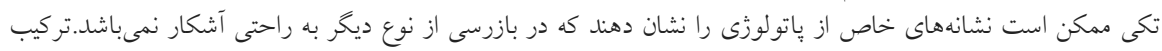

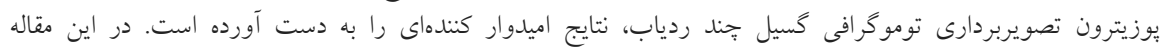

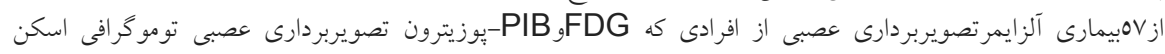

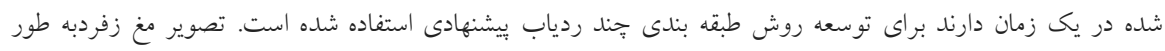

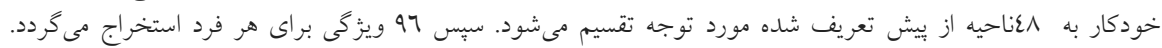

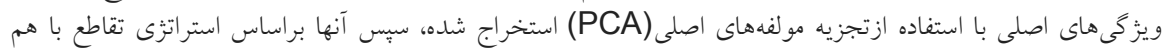

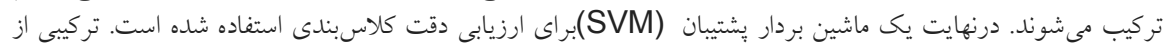

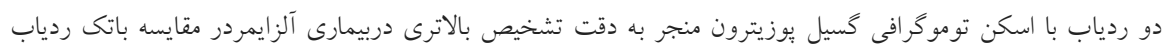
وروشهاى تركيبى ديخرمى شود.

doi: 10.5829/idosi.ije.2014.27.01a.07 


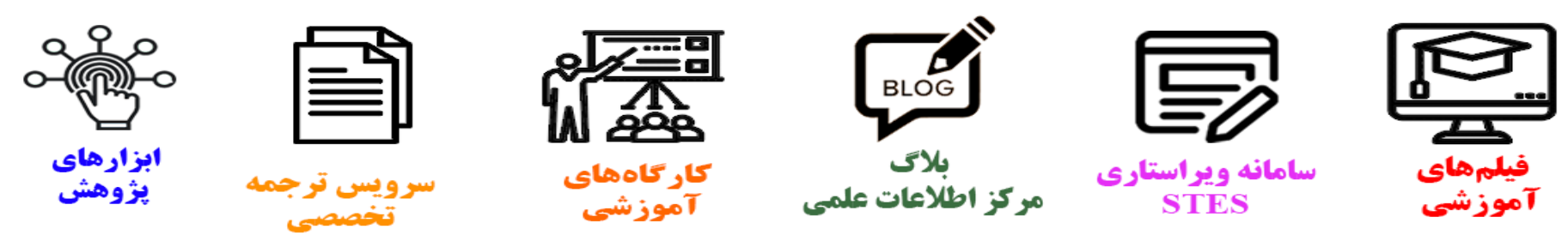

\section{(c)}

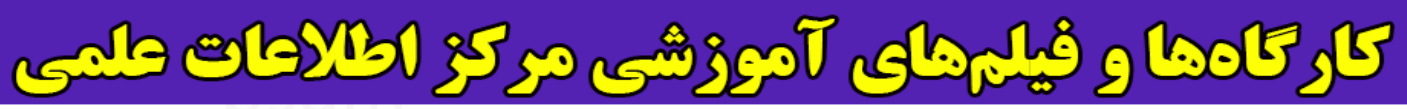
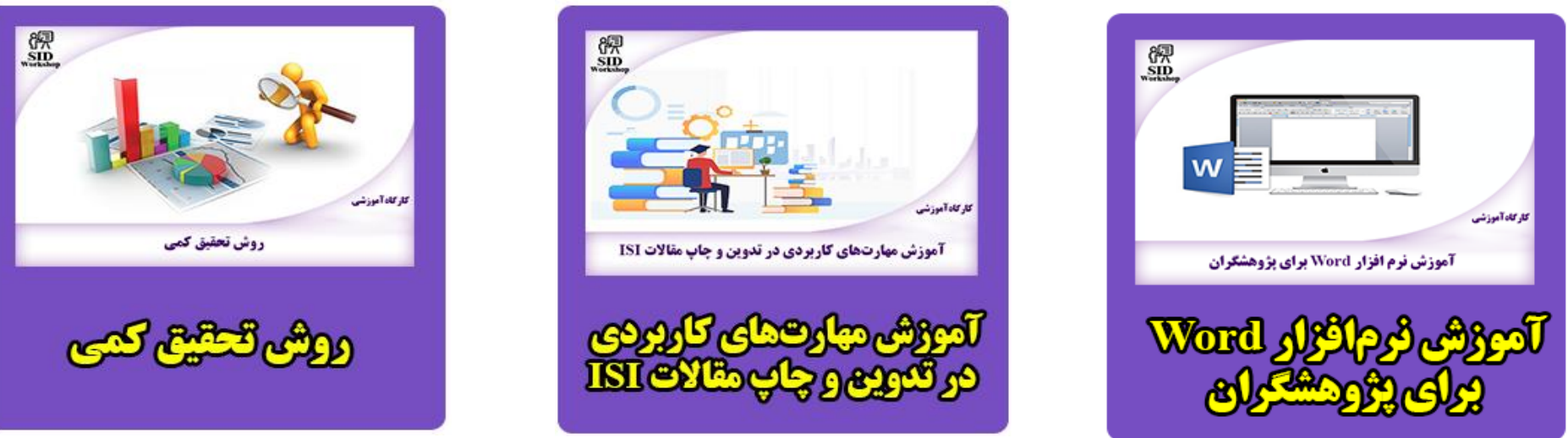